\title{
Dynamics Modeling and Evaluation of Deterministic Dynamics in Advanced Avionics Networks
}

\author{
Guoquan Zhang ${ }^{1}$,Qifeng Ren ${ }^{1, \mathrm{~b}}$, Qiming Yang ${ }^{2}$,Jiandong Zhang ${ }^{2, \mathrm{a}}$,Yan Zhang ${ }^{2}$ \\ ${ }^{1}$ Aeronautical radio electronics research institute of China, Shanghai, 200241, China \\ ${ }^{2}$ Northwestern Polytechnical University, Xi'an, 710072, China \\ aemail: jdzhang@nwpu.edu.cn, bemail: ren_qifeng@careri.com
}

Keywords: advanced avionics network; network dynamics; network resilience; topology optimization

\begin{abstract}
In recent years, the development of avionics system is rapid, and many advanced technologies have been adopted, that improve the performance of avionics system and increase the complexity of system structure.Therefore, it is urgent to optimize the avionics system by studying the avionics network topology and the message transmission mechanism in the system. Based on the theory of complex network and its network dynamics, the network topological structure model of advanced avionics system is established according to engineering practice. The time determinism of this model and the network model are analyzed in random failure mode and malicious attack mode.
\end{abstract}

\section{Introduction}

Modern avionics system is a complex system with complex system structure and management features, which supports simultaneous processing of multiple tasks, simultaneous transmission of multiple information and simultaneous multi- processes. Traditional low-speed bus has been unable to meet the advanced avionics system for the amount of data transmission needs, not to mention the reliability and real-time requirements. At the same time, the avionics system network based on time-triggered Ethernet is the representative of the advanced avionics system.

Based on the complex network dynamics research, this paper studies the avionics system network with time-triggered Ethernet, analyzes the avionics network from the elastic performance of the network, and evaluates the the avionics system network .

\section{Advanced avionics network topology modeling}

\subsection{Complex network characteristic quantities}

(1) Degree distribution

undirected networks: $D_{i}=n, D_{i}$ is the degree of the node $i, n$ indicates the number of nodes adjacent to the node $i$.

directed networks: $D_{i}=D_{i i}+D_{i o}, D_{i i}$ is the number of edges into the node $i, D_{i o}$ is the number of edges leaving the node $i$.

Average degree: $\langle D\rangle=\frac{1}{N} \sum_{i=1}^{N} D_{i}$,represents the mean of the degree of the network. $p(k)$ is the ratio of the number of nodes with degree $k$ in the network to the total number of nodes.

(2) Average shortest path

There is one or more connection paths between any two nodes, in which the path with the least number of nodes is called the shortest path, and the average of all these shortest path lengths is the average shortest path of the network [1].

$G(V, E)$ is the map, based on the set of nodes $V$ and the set of edges $E \subseteq\left\{\left(v_{i}, v_{j}\right): v_{i}, v_{j} \in V\right\}$, 
$l\left(v_{i}, v_{j}\right)$ is the length of the shortest path between any two nodes $v_{i}$ and $v_{j}$, for $G(V, E)$, the average shortest path $L$ is:

$$
L=2 \sum_{v_{i}, v_{j} \in V} l\left(v_{i}, v_{j}\right) / n \times(n-1)
$$

\subsection{Network evolution model based on engineering compromise (TMBE)}

A Two-dimensional region $L_{x} \times L_{y}$, The initial network has $m_{0}$ nodes and $e_{0}$ lines. New node $\boldsymbol{i}\left(x_{i}, y_{i}\right)$,according to certain rules, establish a connection with the existing network nodes, the rules are as follows:

(1) Determine the value of $m$

For the Connection model with a given average degree[i]: we need to know every new node establishes a connection with which existing nodes in the network. Assume that the resulting final network average is $\bar{k}$,

And every new node may be connected with $k \in\{1,2, \ldots, \max k\}$ nodes, $\max k$ is a positive integer. As $k=i$, Let the probability , meet the conditions, $p_{i} i \in\{1,2, \ldots, \max k\}$ :

$$
1 \times p_{1}+2 \times p_{2}+\ldots+\max k \times p_{\max k}=\frac{\bar{k}}{2}
$$

(2) New node connection policy

The new node node $_{m+1}$ establishes the connection to $k$ nodes existing and calculates the properties of the newly generated network.

(1)Delay Normalized Metric of Network, $m_{H i}$

The average shortest path is chosen as the normalized metric of network delay. When node $e_{m+1}$ and node $e_{i}$ establish connection, $i=\{1,2, \ldots, \mathrm{m}\}$. The network delay is $h_{i}$, The minimum delay is $h_{\min }$,The maximum delay is $h_{\max }$. The delay normalized metric of the network is:

$$
m_{H i}=\frac{h_{\max }-h_{i}+\gamma_{H}}{h_{\max }-h_{\min }+\gamma_{H}}
$$

$\gamma_{H}$, as the control parameters.

(2)Normalized Measure of Network Robustness, $m_{R i}$

When node $_{m+1}$ and node $e_{i}$ establish connection, $i=\{1,2, \ldots, \mathrm{m}\}$.The proportion of nodes can still communicate after the network broken is $r_{i}$, The normalized metric of network robustness is:

$$
m_{R i}=\frac{r_{i}-r_{\min }+\gamma_{R}}{r_{\max }-r_{\min }+\gamma_{R}}
$$

$\gamma_{R}$, as the control parameters.

(3)Connection judgment metric measure

Let the cost weight of the network be $\omega_{D}$, Delay weight is $\omega_{H}$, The robustness weight is $\omega_{R}$, Throughput weight is $\omega_{T}$, Then connect the judgment metric measure :

$$
\begin{gathered}
\text { measure }=\omega_{D} m_{D i}+\omega_{H} m_{H i}+\omega_{R} m_{R i}+\omega_{T} m_{T i} \\
\text { s.t. } \omega_{D}+\omega_{H}+\omega_{R}+\omega_{T}=1 \\
\omega_{D} \in[0,1], \omega_{H} \in[0,1], \omega_{R} \in[0,1], \omega_{T} \in[0,1]
\end{gathered}
$$

The new node $_{m+1}$ and the node with the most value of measure create the line.If there are multiple candidate node conditions, then randomly select one to establish a connection with the new node. 


\subsection{Advanced avionics network modeling}

Use the TMBE model to generate a network, as follows:

Step 1: Set the node location information into the node waiting list;

Step 2: At each time step, a joining node is taken out randomly from the node waiting list, and the connection is established with the existing nodes in the network according to the TMBE model rule.

Step 3: Repeat step 2 until the node list is empty.

When avionics network system initial switchboard is 10 groups, suppose the average degree of nodes is $3, \omega_{D}=0, \omega_{H}=0.3, \omega_{R}=0.4, \omega_{T}=0.3$. The node location information is shown in Table 1 .

Table 1 Node location information

\begin{tabular}{r|c|c|c|c|c}
\hline \multicolumn{1}{c|}{ node } & $\mathrm{x}$ & $\mathrm{y}$ & node & $\mathrm{x}$ & $\mathrm{y}$ \\
\hline $\mathrm{A}$ & 2 & 10 & $\mathrm{~B}$ & 7 & 10 \\
\hline $\mathrm{C}$ & 12 & 10 & $\mathrm{D}$ & 17 & 10 \\
\hline $\mathrm{E}$ & 19 & 5 & $\mathrm{~F}$ & 17 & 0 \\
\hline $\mathrm{G}$ & 12 & 0 & $\mathrm{H}$ & 7 & 0 \\
\hline $\mathrm{I}$ & 2 & 0 & $\mathrm{~J}$ & 0 & 5 \\
\hline
\end{tabular}

TMBE model generated using the avionics system network switch topology shown in Figure 1.

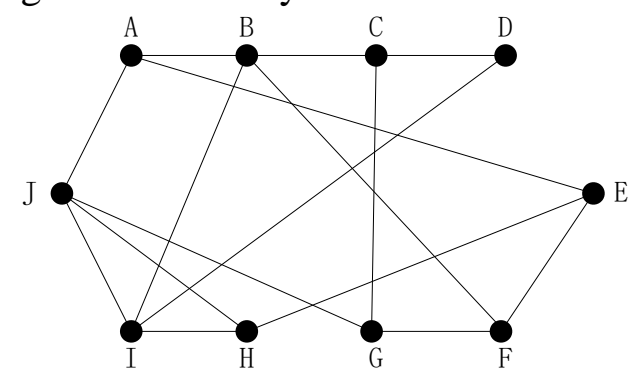

Fig.1 Topological structure of network switch in avionics system

A total of at least 200 terminals in the advanced avionics system network, 200 randomly allocated terminals, the greater the value of the switch node, that the stronger the communication capacity of the switch node, the distribution of the terminal more.The results of terminal assignment are shown in Table2:

Table 2 network topology diagram of the switch node degree distribution

\begin{tabular}{c|c|c|c|c|c}
\hline $\begin{array}{c}\text { Switch } \\
\text { node }\end{array}$ & D & $\begin{array}{c}\text { Terminal } \\
\text { number }\end{array}$ & $\begin{array}{c}\text { Switch } \\
\text { node }\end{array}$ & D & $\begin{array}{c}\text { Terminal } \\
\text { number }\end{array}$ \\
\hline A & 3 & 19 & F & 3 & 19 \\
\hline B & 4 & 25 & G & 3 & 19 \\
\hline C & 3 & 19 & H & 3 & 19 \\
\hline D & 2 & 12 & I & 4 & 25 \\
\hline E & 3 & 19 & J & 4 & 25 \\
\hline
\end{tabular}

\section{Elasticity analysis:}

\subsection{The concept of flexibility in avionics network}

In the avionics network, we main concern network on failure.The elasticity of the system is measured by the extent to which the system absorbs the damage..After the destruction of the information network, its function or performance will be changed, the destructive force as an independent variable, the function or performance change as a variable from the size of the variable can represent the degree of damage to the network absorption level,The amount of change for the network or performance.

1、Avionics network efficiency

Avionics network efficiency is defined as $E=\frac{1}{n(n-1)} \sum_{i \neq j} \frac{1}{l_{i j}}$, The average of the sum of the 
shortest distances between the two nodes, which represents the ease of avionics average communication.

2.Node vulnerability

The vulnerability of a node is:

$$
V_{i}=\frac{E-E_{i}}{E}
$$

$E_{i}$ Is the network efficiency after removing the node $i$ from the network.

\subsection{Elasticity analysis of avionics network}

The network dynamics analysis of avionics system is mainly to analyze the network performance in random failure mode and malicious attack mode. In this paper, the background of random failure mode is selected as the node failure, while the malicious attack mode is chosen based on the degree and the number of attacks, while increasing the vulnerability based on the node to compare the network function to measure network efficiency and S-elasticity, compare with their applicability.

The communication between the avionics system network system depends on the topological structure between the switches. Therefore, only the switch topology is considered when calculating the efficiency and S-elasticity of the network.

1. Establishment of stochastic failure model

The advanced avionics system network generates random failures using the following fault model assumptions:

Without considering the weights of each node in the advanced avionics system network model, the fault node in the advanced avionics system network model is randomly generated with probability p;

The faulty node and the edge of the node connected to other nodes are deleted by themselves.

2.Random fault simulation

Fault simulation experiment according to the above fault strategy. The simulation process of random failure model is as follows:

1) Determine the number of switch node $n$, the establishment of switch node coordinate matrix Node and the switch node connection matrix $A$, the rules are as follows:

$$
A_{i j}=\left\{\begin{array}{c}
1, i \neq j \text { (connected }) \\
0, i \neq j(\text { unconnected }) \\
\infty, i=j
\end{array}\right.
$$

2) Randomly generates the fault terminal node, sets the corresponding connection property, and draws the topology diagram of the network model;

3) to determine the probability of random failure, and find the nodes resulting in random fault node;

4) Determine the switch connection matrix and the faulty terminal connection attributes after the fault;

5) Drawing the network model after the random fault ,and calculate the elasticity of the network under stochastic failure.

Figure 2 shows the network topology before the fault. 


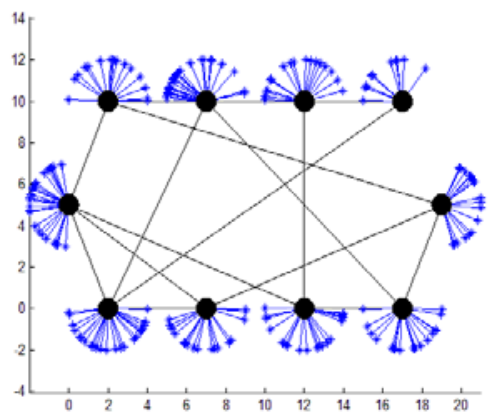

Figure 2 Advanced avionics system network topology simulation diagram

From the definition of network efficiency, it is necessary to compute the shortest path between nodes. Network efficiency when there is no network failure:

Compute the network S-elasticity:

$$
E=\frac{1}{n(n-1)} \sum_{i \neq j} \frac{1}{l_{i j}}=0.589
$$

$$
R_{S}=\frac{\tau(G-S)}{n} \times \frac{|S|+\tau(G-S)}{l(G-S)}
$$

Average shortest path:

$$
\begin{gathered}
L=\frac{2 \sum_{v_{i}, v_{j} \in V} l\left(v_{i}, v_{j}\right)}{n \times(n-1)} \\
l(G-S)=\frac{1}{10 \times 9}(7+5+7+7+3+3+2) \times 2=\frac{1}{90} \times 68=0.756
\end{gathered}
$$

The system network S-elasticity without failure is :

$$
R_{S}=\frac{\tau(G-S)}{n} \times \frac{|S|+\tau(G-S)}{l(G-S)}=\frac{10}{10} \times \frac{0+10}{68} \times 90=13.235
$$

The model of Fig 3,a random fault occurs according to the probability of $5 \%, 15 \%$, the simulation results are as follows:

1) Random failure probability $p=5 \%$, and randomly delete 11 nodes.

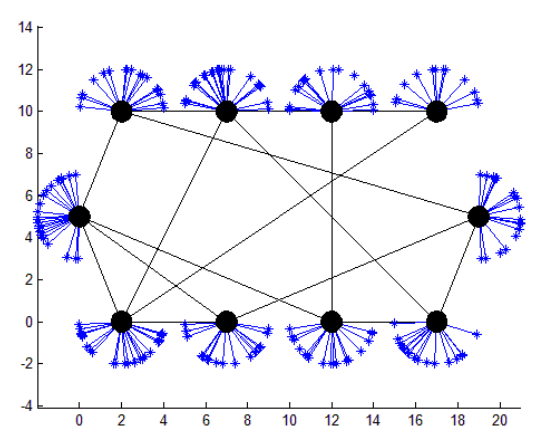

Fig.3 Simulated graph of network topology when the probability of random failure is $5 \%$

It can be seen from Figure 3, with a probability of 5\% of random failure, the switch does not change the topology,Can be approximated that does not have an impact on network performance,At this point the advanced avionics system network efficiency is still 0.589, the network's S-performance is still 13.235

2) Random failure probability $p=15 \%$, and randomly delete 32 nodes.

When the stochastic failure probability is $15 \%$, the network topology simulation is shown in Fig 4. The network efficiency is 0.444 and the S-performance of the network is 10.125. 


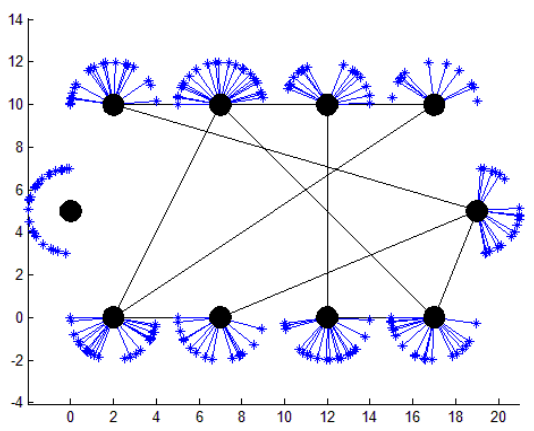

Fig.4 Simulated graph of network topology when the probability of random failure is $15 \%$

As a result, the network efficiency of the advanced avionics system network decreases and the S-elasticity of the network decreases as the number of nodes / nodes increases.This means that as the number of nodes / nodes increases, the communication becomes more and more difficult, and the function of the remaining network becomes weaker and weaker.

\section{Concluding remarks}

The avionics system is developing in the direction of "advanced", "comprehensive" and "networked". As the requirement of avionics system becomes higher and higher, the system becomes more and more complicated.And real-time requirements more stringent, the use of a new generation of aviation data bus will become the inevitable trend of the development of avionics systems.In order to study the topological structure and performance parameters of the advanced avionics system network using TTE bus, this paper combines the theory of complex network and dynamics, from the angle of theory and engineering practice, the network of advanced avionics system is studied,The dynamic performance of the model: Elastic properties.

At present, the research on advanced avionics system is very limited by complex network theory and network dynamics theory. Therefore, this paper has a certain reference value for the research of advanced avionics system dynamics in the future.

\section{Acknowledgement}

The work was supported by the Science and Technology on Avionics Integration Laboratory. The gratitude goes to all the staff of the Foundation.

\section{References}

[1]Lei Feng,Yuguang Zhang,Li Tang. The Application of Complex Network Theory in Personalized Recommendation Service of Library[J]. Intelligence Theory and Practice,2009,02:69-71.

[2]Dongjie Zhao,Yu He,Haitao Yang,Hua Wang,Zhi Li,Hongli Zhao. Design Optimization of Complex Information Network Based on Evolution[J]. Science \& Technology Review,2011,36:23-27.

[3]FERRER R,SOLE R V. Optimization in Complex Networks[J]. Lect. Notes Phys. 2003,25(6):114-125.

[4]BING WANG, HUANGWEN TANG, CHONGHUI GUO, ZHILONG XIU. Entropy optimization of scale-free networks' robustness to random failures[J]. Physica A. 2006,363: 591-596.

[5] Haibao Tian, Wentian Gao, Yong Wu, Jiandong Zhang. Performance evaluation of the Integrated Avionics Simulation system based on grey theory[C]. 2011 IEEE 3rd International Conference on Communication Software and Networks, ICCSN 2011, p 56-59. 